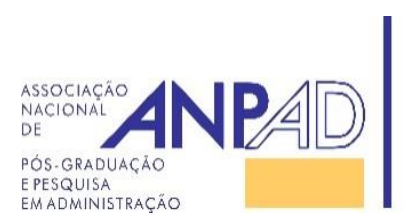

Disponível em
http://www.anpad.org.br/rac
RAC, Rio de Janeiro, v. 20, n. 1, art. 2,
pp. 21-40, Jan./Fev. 2016
http://dx.doi.org/10.1590/1982-7849rac20161842

$(\mathrm{cc}) \mathrm{EY}$

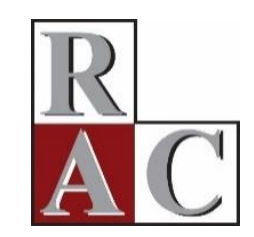

\title{
Impacto Social: Estudo sobre Programas Brasileiros Selecionados de Pós-graduação em Administração de Empresas
}

Social Impact: A Study of Selected Brazilian Graduate Management Programs

Thomaz Wood Jr. ${ }^{1}$

Caio César Medeiros Costa ${ }^{1}$ Giovanna de Moura Rocha Lima ${ }^{1}$

Rosana Córdova Guimarães ${ }^{1}$

Fundação Getulio Vargas - Escola de Administração de Empresas de São Paulo ${ }^{1}$

Artigo recebido em 20.02.2014. Última versão recebida em 18.04.2015. Aprovado em 20.04.2015. 


\title{
Resumo
}

Existe, na comunidade acadêmica, uma crescente preocupação com a questão do impacto social: os benefícios que as instituições de ensino e pesquisa trazem para a sociedade. Este artigo tem como objetivo contribuir para o debate sobre o impacto social no âmbito dos programas brasileiros de pós-graduação em Administração de Empresas. Para isso, este texto toma por base a literatura científica sobre impacto social e apresenta um estudo exploratório que envolveu três dimensões de impacto social: (a) a formação de pesquisadores em programas de doutorado, considerando os egressos dos programas e sua inserção no mercado de trabalho; (b) a utilização do conhecimento gerado pelos pesquisadores de cada programa, medido pelo fator $\mathrm{H}$ institucional, com a utilização do software Publish or Perish (PoP) sobre a base do Google Scholar; e (c) a citação de pesquisadores e de seus estudos na mídia especializada em negócios, medida por meio de um levantamento que tomou por base artigos veiculados nas publicações de negócios mais relevantes do Brasil. Essas três dimensões foram avaliadas em quatro programas de pós-graduação em Administração: FEA-USP, FGV-EAESP, PPGA-UFRGS e CEPEAD-UFMG. Os resultados indicam diferenças substantivas entre os programas nas três dimensões, favorecendo os mais antigos e localizados em São Paulo. Conclui-se que há, nas três dimensões pesquisadas, oportunidades para aumentar o impacto social. Sugerem-se ações para atender esse objetivo e procura-se indicar caminhos para desenvolver o conhecimento sobre o impacto social no âmbito dos programas brasileiros de pós-graduação em Administração de Empresas.

Palavras-chave: impacto social; pós-graduação em administração de empresas; mídia; doutorado; fator H.

\begin{abstract}
In the academic community there is a growing concern with the issue of social impact: the benefits that teaching and research institutions bring to society. This paper seeks to contribute to the debate on the social impact Brazilian graduate management programs have. It is based upon scientific literature on social impact and presents an exploratory study of three social impact dimensions: (a) the training of researchers in doctoral programs, focusing on graduates and their insertion in the labor market; (b) the use of knowledge generated by each program's researchers, as measured by the institutional $\mathrm{H}$ factor, using the Publish or Perish (PoP) software to survey the Google Scholar database; and (c) the citation of researchers and their studies in business media, measured through a survey of articles published in the most relevant Brazilian media outlets. These three dimensions were measured in four graduate programs: FEA-USP, FGV-EAESP, PPGA-UFRGS and CEPEAD-UFMG. The results show significant differences among the programs, favoring the oldest ones, which are located in São Paulo. The conclusion is that there are opportunities to improve social impacts in these three dimensions and recommended measures are suggested to help meet this goal. The study also indicates directions to develop further knowledge about what social impacts Brazilian graduate management programs can have.
\end{abstract}

Key words: social impact; graduate management programs; media; $\mathrm{PhD} ; \mathrm{H}$ factor. 


\section{Introdução}

No dia 26 de dezembro de 2013, o médico Marco Antonio Zago foi escolhido pelo governador de São Paulo, Geraldo Alckmin, a partir de uma lista tríplice, para o cargo de reitor da Universidade de São Paulo (USP). Durante a campanha, Zago declarou que "a USP precisa fazer mais. Isso porque, além de centro produtor de conhecimento e de formação, a USP tem que ser instrumento de mudança da sociedade" (O Estado de S. Paulo, 2013).

As palavras de Zago são significativas e vêm ao encontro das expectativas, declaradas pelo governador do Estado, de que a USP deve apoiar o desenvolvimento de políticas públicas (Vieira, 2013). De fato, existe, no mundo acadêmico, uma crescente preocupação com o impacto social, ou seja, em gerar conhecimento e realizar atividades capazes de trazer benefícios para a sociedade. Assim, a ciência deve ser vista como um amplo sistema social, o qual deve ter várias funções, entre as quais se encontra a disseminação do conhecimento (Macias-Chapula,1998).

Entretanto, a avaliação e a mensuração do impacto social do conhecimento gerado por instituições de ensino e pesquisa não é tarefa trivial. Até o momento, apesar de diversos estudos e avaliações terem sido realizados, parece não ter sido consolidada uma metodologia para a medição do impacto social (Comissão Europeia, 2010; Garrett-Jones, 2000; Guisado, Cabrera, \& Cortés, 2010; PalomaresMontero, García-Aracil, \& Castro-Martínez, 2008; The Association to Advance Collegiate Schools of Business [AACSB], 2008). Isso decorre, entre outros fatores, da alta complexidade da tarefa, fruto da diversidade dos sistemas de ensino e pesquisa, das diferenças entre campos científicos (entendidos como áreas de conhecimento e espaços de interação social) e de dificuldades associadas à própria operacionalização da medição (Walker, Alexander, Barros, Cochrane et al., 2011; Walker, Alexander, Barros, Guzman et al., 2011).

Esta pesquisa insere-se no contexto da avaliação dos programas de pós-graduação stricto sensu no Brasil. De fato, o sistema vigente no Brasil contempla, como um de seus cinco critérios, a inserção social, a qual inclui a formação de docentes, ou seja, recursos humanos que possam contribuir para o aprimoramento da gestão pública e a redução da dívida social, para o desenvolvimento cultural e artístico, e para o desenvolvimento microrregional, regional ou nacional (Coordenação de Aperfeiçoamento de Pessoal de Nível Superior [CAPES], 2013). O critério de inserção social também destaca questões relacionadas à disseminação do conhecimento, inclusive por meio de entrevistas e matérias em jornais.

Este artigo tem como objetivo contribuir para o debate sobre o impacto social no campo do ensino e pesquisa da Administração de Empresas no Brasil. Adotaram-se, como foco de estudo, quatro programas brasileiros de pós-graduação, aqueles que obtiveram notas 6 ou 7 pelo sistema de avaliação da CAPES no triênio 2007-2009, baseados na FGV-EAESP, na FEA-USP, no PPGA-UFRGS e no CEPEAD-UFMG.

O texto está dividido em seis seções, incluindo esta introdução. A segunda seção define impacto social e indica um modelo simplificado para sua medição no âmbito dos programas brasileiros de pósgraduação em Administração, além de apresentar brevemente as discussões relacionadas ao tema no âmbito das escolas de negócios. A terceira seção trata dos procedimentos metodológicos adotados para as três frentes de investigação realizadas, respectivamente relacionadas aos impactos relativos: à formação de pesquisadores, à ciência e à repercussão na mídia. A quarta seção apresenta os resultados das três frentes de investigação, em subseções dedicadas a cada um dos três tipos de impacto mencionados anteriormente. A quinta seção apresenta uma discussão dos resultados e adota uma perspectiva propositiva, indicando caminhos para aumentar o impacto social do conhecimento. A sexta seção explicita as contribuições do artigo e sugere caminhos para futuras pesquisas. 


\section{Impacto Social do Conhecimento}

Esta seção propõe uma definição para o conceito de impacto social do conhecimento e apresenta um modelo para análise e avaliação do impacto social do conhecimento, sugerindo uma adaptação para uso no presente estudo. Além disso, procura situar a questão do impacto social do conhecimento nos debates e esforços realizados no âmbito das escolas de negócios.

A ideia que fundamenta o conceito de impacto social é a de apropriação e utilização do conhecimento pela sociedade (Lima \& Wood, 2014). O relatório Science, the Endless Frontier (Bush, 1945) estabeleceu o princípio segundo o qual a ciência deve responder às necessidades da população, constituindo marco inicial para reflexões e desenvolvimentos sobre a questão do impacto social do conhecimento.

Nos últimos anos, diversas iniciativas foram tomadas visando avaliar o impacto social de instituições de ensino e pesquisa sobre a sociedade (Comissão Europeia, 2010; Garrett-Jones, 2000; Guisado et al., 2010; Palomares-Montero et al., 2008). Em 2008, um grupo-tarefa constituído no âmbito da AACSB, principal organização certificadora de escolas de negócios, publicou amplo e bem fundamentado estudo sobre a questão do impacto da pesquisa e do conhecimento gerado por escolas de negócios (AACSB, 2008). O relatório desse grupo-tarefa foi posteriormente tomado como base para a definição de diretrizes (AACSB, 2012) e padrões (AACSB, 2013) para instituições de ensino e pesquisa certificadas pela AACSB em todo o mundo. Os autores do relatório (AACSB, 2008) argumentam que contribuições intelectuais podem se materializar de várias formas e atingir diferentes propósitos, incluindo pesquisas científicas de base, que visam avançar o conhecimento sobre a gestão; pesquisas aplicadas, que têm como objetivo prover soluções para problemas reais; e pesquisas sobre aprendizagem e pedagogia, visando aperfeiçoar métodos de aprendizagem, solução de problemas e desenvolvimento de habilidades.

Propõe-se, então, com base no referencial analisado, definir impacto social para o propósito específico deste estudo como o benefício recebido por indivíduos ou grupos de indivíduos, por uma organização (por exemplo, uma empresa privada ou pública, ou uma organização social), por um setor de atividades (por exemplo, uma cadeia produtiva), por um campo científico (por exemplo, o campo de Estudos Organizacionais ou o campo de Gestão da Tecnologia), ou, ainda, pela sociedade em geral, originado por processos relacionados à geração ou disseminação do conhecimento, e ao ensino, realizados no âmbito de instituições de ensino e pesquisa.

No âmbito das instituições de ensino e pesquisa em Administração, o conhecimento ou contribuição intelectual pode ser gerado por meio da pesquisa científica. No entanto, cabe observar que a contribuição intelectual não se restringe às publicações realizadas em periódicos científicos. A pesquisa científica realizada nas instituições de ensino e pesquisa em Administração relaciona-se a atividades planejadas para prover respostas a questões que possuem importância prática ou teórica, o que inclui análise do comportamento nas organizações e do contexto social e econômico que as envolve (AACSB, 2008).

\section{Modelo para analisar e avaliar o impacto social do conhecimento}

Um modelo para análise e avaliação do impacto social do conhecimento pode ser retrospectivo, realizado por meio da reconstrução história da sequência ou cadeia de processos de conhecimento e seus efeitos, ou prospectivo, estabelecendo-se as possibilidades de uso do conhecimento ou as consequências da difusão e uso de certas tecnologias no início de um projeto de pesquisa (Estébanez, 2003). Pode-se pensar, ainda, em uma avaliação in itinere (Guisado et al., 2010), feita quando o projeto ou programa está em curso e em um nível avançado de realização. Esse tipo de avaliação destina-se a assegurar o acompanhamento das ações implantadas, para confrontar com os impactos projetados. No presente estudo exploratório, foi realizada uma avaliação retrospectiva, ou seja, baseada em fatos e indicadores passados. 
No campo da Administração de Empresas, como em outras ciências sociais, a avaliação do impacto social do conhecimento é especialmente desafiadora, pois raramente envolve patentes, protótipos, produtos ou artefatos que podem ser medidos de maneira objetiva e direta. Com isso, a avaliação pode ficar restrita a medidas indiretas. A AACSB (2013), por exemplo, apresenta uma extensa lista de métricas de impacto. A maioria dos itens corresponde a medidas indiretas, tais como: publicações em periódicos qualificados, contagem de downloads de artigos, prêmios por pesquisas, incorporação de artigos científicos em syllabi de cursos, prêmios por trabalhos científicos, citações na mídia, relatórios de consultoria e estudos de casos, participações em seminários, acesso a verbas de agências de fomento, etc. Alguns itens, entretanto, correspondem a medidas diretas, que podem ser relacionadas a mudanças substantivas na realidade, tais como a adoção, por organizações, de novas práticas e abordagens operacionais e a participação de especialistas na formulação de políticas públicas.

Portanto, neste ponto, a questão que se coloca é: que indicadores medir? Santos (2003) utilizou uma abordagem desenvolvida pelo Centro de Sociologia da Inovação da Escola MINES ParisTech, fundada no princípio de que a pesquisa e a produção acadêmica constituem uma atividade cuja natureza e resultados podem ser analisados e avaliados segundo cinco dimensões: (a) formação, relacionada ao sistema de ensino; (b) geração de inovações, relacionada ao mercado; (c) presença na mídia, relacionada à disseminação do conhecimento; (d) políticas públicas, relacionadas à influência sobre diretrizes de governo; e (e) conhecimentos certificados, relacionados à comunidade científica.

A abordagem proposta por Santos (2003) antecipa a conceituação pluralística defendida por Aguinis, Shapiro, Antonacopoulou e Cummings (2014). Tais autores criticam a abordagem usual de avaliação acadêmica, que se baseia somente em um grupo de interesse (os próprios acadêmicos). Eles afirmam que tal abordagem é restrita e insuficiente. Em seu lugar, propõem a adoção de um quadro conceitual amplo de avaliação do impacto. Entretanto, Aguinis et al. (2014) apenas sugerem o modelo conceitual geral e listam indicadores possíveis, sem definir categorias, enquanto Santos (2003) indica explicitamente categorias e indicadores.

O modelo de Santos (2003) reconhece a existência da diversidade da produção acadêmica e dos diferentes aspectos sociais que os seus resultados podem influenciar. Tal modelo pode ser considerado, portanto, um ponto de partida completo e abrangente para este estudo, o qual utilizará três das cinco dimensões propostas por Santos (veja Figura 1): a primeira, relacionada à formação de doutores; a terceira, relacionada à presença na mídia; e a quinta, relacionada aos conhecimentos apropriados pela própria comunidade científica.

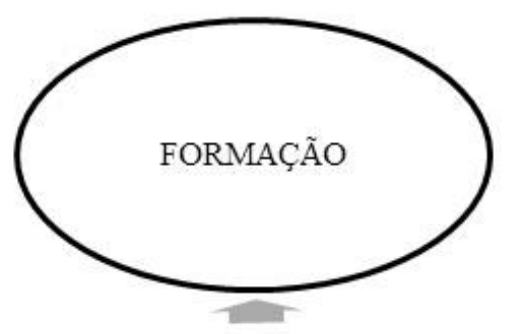

IMPACTO SOCIAL DO

CONHECIMENTO
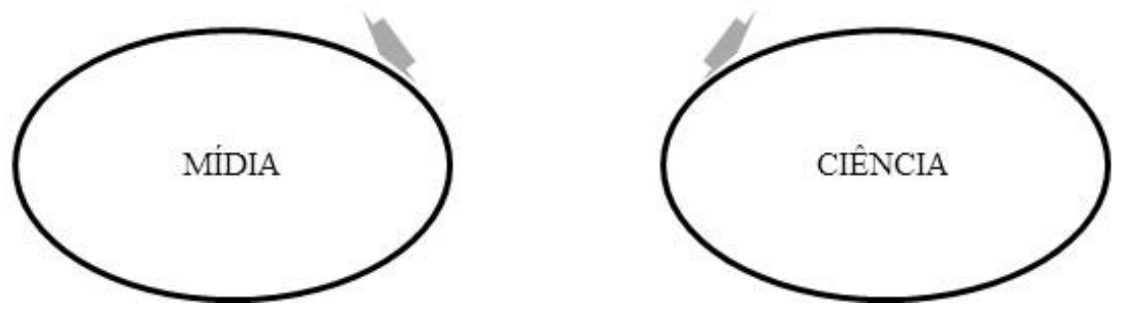

Figura 1. Modelo Simplificado de Impacto Social do Conhecimento 
Cabe observar que a primeira dimensão refere-se à formação, enquanto a terceira e a quinta dimensão referem-se à disseminação do conhecimento. No entanto, as duas últimas devem ser mantidas separadas, como no modelo original de Santos (2003), por tratarem de processos distintos: a terceira dimensão relaciona-se à disseminação do conhecimento para a comunidade em geral, e a quinta relaciona-se à disseminação do conhecimento especificamente para a comunidade científica.

A segunda dimensão - geração de inovações - não foi pesquisada devido às dificuldades inerentes à avaliação do impacto da pesquisa em Administração de Empresas sobre as práticas gerenciais, e à inexistência, pelo menos ao conhecimento dos autores do presente estudo, de métodos consistentes para realizar tal avaliação. A quarta dimensão - políticas públicas - não foi pesquisada por não constituir objeto prioritário do campo da Administração de Empresas, embora alguns tipos específicos de estudos, relacionados, por exemplo, a questões tais como cadeias produtivas, produtividade e administração da ciência e tecnologia, possam gerar impactos sobre políticas públicas. Apesar dessas limitações, considera-se que o modelo apresenta abrangência satisfatória para um trabalho exploratório sobre o tema. Serão apresentados, a seguir, os métodos de investigação e os resultados das três dimensões investigadas.

\section{Debates e esforços no âmbito das escolas de negócios}

No âmbito das escolas de negócios, as discussões em torno do impacto social do conhecimento podem ser relacionadas à onda de críticas ocorrida a partir dos anos 2000. Em um artigo polêmico - The end of business schools? Less success than meets the eye, Pfeffer e Fong (2002) questionaram diretamente a falta de aplicabilidade prática da pesquisa realizada nas escolas de negócios, além de colocar em xeque o impacto dos MBAs na carreira profissional.

Diversos outros autores seguiram a trilha aberta por Pfeffer e Fong (2002). Bennis e O'Toole (2005) criticaram o afastamento das escolas de negócios em relação às necessidades das empresas. Rubin e Dierdorff (2009) revelaram, por meio de uma pesquisa empírica, que os MBAs não atendiam, em suas disciplinas, as competências gerenciais mais requeridas pelas organizações. Mintzberg (2004), por sua vez, defendeu argumento contundente, segundo o qual não se pode formar gestores em sala de aula.

Por outro lado, autores filiados à corrente crítica dos estudos de gestão (Chia, 1996, 1999; Grey \& French, 1996; Grey, Knights, \& Willmott, 1996; Reynolds, 1997; Willmott, 1994) vinham estabelecendo, desde a década de 1990, posição contra a transformação dos programas de gestão em mercadorias; contra a visão simplista e meramente instrumental do conhecimento em gestão, permeado por modelos prontos e receitas; e contra a tentativa de transformar os estudantes em meros espectadores, sem autonomia ou consciência crítica.

Em resposta ao ambiente crítico, a AACSB deu início a uma série de estudos e esforços voltados para apoiar as instituições certificadas na promoção do impacto social. Conforme citado anteriormente, um grupo de trabalho formado na segunda metade da década de 2000 focalizou especificamente a questão do impacto social da pesquisa (AACSB, 2008).

Os autores do estudo da AACSB reconhecem a ênfase excessiva dada à pesquisa básica e à publicação em periódicos científicos e o foco insuficiente na pesquisa aplicada e no ensino. Como alternativa, oferecem uma lista não exaustiva de contribuições científicas, classificadas em três categorias (AACSB, 2008, p. 17), relacionadas às contribuições: (a) para a ciência; (b) para a prática; e (c) para o ensino e o aprendizado.

Em documentos posteriores (AACSB, 2012, 2013), a mesma associação identificou indicadores em diversas dimensões relacionadas ao impacto social. A lista foi elaborada com base em um estudo exploratório com escolas de negócios. Os autores observam que a lista não tem a pretensão de ser completa e não deve ser vista como recomendação para indicadores específicos. Entretanto, sinalizam claramente a importância de orientar esforços para o atendimento de objetivos relacionados ao impacto 
social. São apresentadas, a seguir, a título de ilustração, algumas categorias de impacto e alguns exemplos de indicadores:

Impacto sobre a prática ou a comunidade: citações na mídia, relatórios de consultoria, apresentações, participação em conselhos de direção de empresas e publicações em revistas profissionais;

Impacto acadêmico: publicações em periódicos científicos escolhidos, contagem de citações, número de downloads de artigos científicos, editoria de periódicos científicos e uso de recursos de agências de fomento;

Impacto na formação de doutores: doutores contratados, publicações realizadas por estudantes e exestudantes, participação em eventos científicos e bolsas de estudos obtidas para estudantes;

Impacto no ensino e aprendizado: estudos de caso que levam à adoção de novas práticas de ensino e aprendizado, livros-texto e manuais, softwares para ensino e aprendizado e estudos de casos;

Centros de estudos: convites de órgãos de governo para participação no desenvolvimento de políticas públicas, uso de recursos de agências de fomento, número de visitas em websites e número de participantes em eventos promovidos pelos centros de estudos.

Naturalmente, a mensuração desses indicadores é complexa e pode esbarrar em dificuldades operacionais, além de apresentar complicações relacionadas a como avaliar os resultados ao longo do tempo para uma dada instituição ou como comparar diferentes instituições. Ainda assim, constitui um leque rico e amplo de possibilidades, que levam a perceber a função social das instituições de ensino e pesquisa de uma forma mais ampla. As categorias e indicadores acima listados são coerentes com o Modelo Simplificado de Impacto do Conhecimento (Figura 1), proposto neste estudo. Pode-se afirmar que a aplicação de tal modelo representa uma tentativa pragmática e exploratória para expandir os meios de avaliação das escolas de negócios e, especificamente, de seus programas de pós-graduação em Administração de Empresas.

\section{Procedimentos Metodológicos}

Esta pesquisa foi desdobrada em três frentes de investigação, correspondendo cada uma delas respectivamente aos impactos relativos à formação de pesquisadores, à ciência e à repercussão na mídia. A escolha dos programas foi baseada na avaliação conduzida pela CAPES no triênio 2007-2009, tomando-se programas com notas 7 e 6 , as mais altas atribuídas pelo sistema. O uso desse critério de escolha pressupõe que o sistema de avaliação, com suas múltiplas métricas, constitui um proxy adequado para a importância e o grau de excelência dos programas. Procurou-se, dessa forma, identificar os programas que, em função de sua avaliação por meio do sistema CAPES, tivessem, entre todos os programas locais, maior potencial para geração de impacto social. Conforme indicado na introdução deste artigo, os programas escolhidos foram os seguintes: CEPEAD-UFMG, FEA-USP, FGV-EAESP e PPGA-UFRGS. Cabe observar que são programas tradicionais, ligados a instituições de projeção nacional, e estão entre os programas com maior número de docentes permanentes e entre os programas que mais produziram teses de doutoramento, considerando os dados da CAPES (2013).

A avaliação do triênio 2007-2009 foi adotada como base por ser a avaliação disponível no período no qual a maior parte do estudo foi realizada. Cabe observar que essa base não foi alterada quando ocorreu a divulgação dos resultados da avaliação do triênio 2010-2012, principalmente pela possibilidade de mudanças nos resultados da avaliação em função de eventuais recursos impetrados pelos programas. Os procedimentos metodológicos para essas três frentes de investigação são apresentados a seguir. 


\section{Procedimentos metodológicos da investigação relacionada à formação de pesquisadores}

A formação de pesquisadores constitui atividade essencial dos programas de pós-graduação. Tal atividade é avaliada trienalmente pelo sistema CAPES, o qual monitora: (a) a quantidade de teses e dissertações defendidas, em relação ao corpo docente permanente e à dimensão do corpo discente; (b) a distribuição das orientações das teses e dissertações defendidas em relação aos docentes do programa; (c) a qualidade das teses, das dissertações e da produção de discentes autores; (d) a eficiência do programa na formação de mestres e doutores bolsistas, considerando o tempo de formação de mestres e doutores, assim como o percentual de bolsistas titulados; e (e) a participação dos discentes e egressos em eventos alinhados à proposta do programa (CAPES, 2013).

É a partir das atividades de ensino que conhecimentos e práticas elaboradas e sistematizadas por pesquisadores são transformados em competências dos alunos que, por sua vez, os colocam em prática nos diferentes setores de atividade: indústria, serviços públicos, ensino e pesquisa (Santos, 2003). No caso dos programas de pós-graduação, cujo foco é a formação de professores e pesquisadores, o impacto social decorre diretamente da ação daqueles que conduzirão novas pesquisas, ensinarão novos alunos em programas de graduação e de pós-graduação e, além disso, liderarão mudanças em organizações, como funcionários, empresários ou consultores. Esse impacto terá maior alcance se houver dispersão geográfica dos egressos.

Para este estudo exploratório, tomou-se como foco o número de doutores egressos no período de 2008 a 2012 e sua inserção no mercado de trabalho. A escolha dos doutores deveu-se a esse extrato constituir o nível mais avançado de formação regular de pesquisadores. Foram identificados os doutores egressos dos quatro programas pesquisados e foram analisadas suas informações registradas na Plataforma Lattes, nos meses de agosto a outubro de 2013. Além do número de egressos por programa, procurou-se também identificar quantos doutores atuam na instituição de origem, quantos seguiram carreira acadêmica e quantos seguiram carreira empresarial, e quantos do primeiro grupo atuam em instituições com nota 6 ou 7 pelo sistema CAPES, considerando a avaliação do triênio 2007-2009. Os resultados são apresentados na seção Primeira dimensão: formação de pesquisadores.

\section{Procedimentos metodológicos da investigação relacionada ao impacto sobre a ciência}

Os primeiros estudos sobre o impacto da produção científica sobre a própria ciência ocorreram há quase 90 anos, com o trabalho pioneiro de Gross e Gross (1927). Com o tempo, as citações foram adotadas como um proxy da qualidade e, consequentemente, do impacto do conhecimento gerado. As citações passaram, então, a ser a principal forma de se medir a dimensão qualitativa da produção científica. $\mathrm{O}$ argumento que sustenta essa prática é simples: quanto mais um trabalho científico é citado, maior é sua influência sobre os pares e também maior a sua qualidade, já que teria sido aceito e validado por um maior número de membros da comunidade acadêmica (Glänzel, 2008).

Assim, as citações e o fator de impacto (FI) se configuraram como a principal forma para a medição da qualidade e da influência da produção científica. Seu uso mais corriqueiro refere-se aos periódicos científicos (Garfield, 1999). Entretanto, apesar da ampla aceitação, o FI é alvo de críticas, frequentemente relacionadas à definição da base de dados e ao método de apuração (Ruiz, Greco, \& Braile, 2009), à limitação da cobertura e à não inclusão de livros, anais e documentos de trabalho, além de não serem numerosos os documentos em línguas diferentes do inglês (Harzing \& Wal, 2008; Sanderson, 2008).

Uma alternativa ao FI e que está se tornando cada vez mais popular é o índice $\mathrm{H}$, proposto originalmente por Hirsch (2005) como uma medida para a avaliação da produção de um único pesquisador (Bornmann \& Daniel, 2007). No entanto, sua utilização pode ir além dessa aplicação e ser realizada em um nível mais amplo de análise, por exemplo, o impacto da produção de grupos de pesquisa (Raan, 2006) ou de periódicos (Braun, Glänzel, \& Schubert, 2006). De fato, o índice H é apontado como um indicador que incorpora medidas de quantidade e de qualidade (Bornmann \& Daniel, 2007; Raan, 2006). 
Segundo Hirsch (2005), o índice H deve ser calculado tomando-se o número de artigos publicados e o número de citações recebidas pelo pesquisador. Assim, um pesquisador possui um índice $\mathrm{H}$ se $\mathrm{H}$ de seus $\mathrm{N}$ artigos tiverem pelo menos $\mathrm{H}$ citações cada um e os outros $(\mathrm{N}-\mathrm{H})$ artigos não tiverem mais que $\mathrm{H}$ citações cada. Por exemplo, se um pesquisador possui um índice $\mathrm{H}$ igual a 10, então os seus 10 artigos mais citados têm no mínimo 10 citações cada e os demais artigos do pesquisador não possuem mais de 10 citações cada. Um procedimento similar é realizado para o cálculo do índice $\mathrm{H}$ institucional. Nesse caso, utilizado no presente estudo, analisam-se em grupo os artigos de todos os professores de cada programa, excluindo-se as repetições.

Este estudo exploratório envolveu a mensuração do impacto da produção científica de professores que compõem os Núcleos Docentes Permanentes (NDPs) dos programas de pós-graduação em Administração de Empresas anteriormente mencionados, por meio do índice $\mathrm{H}$ institucional. As informações sobre os programas e os respectivos NDPs foram levantadas nos websites de cada instituição em agosto de 2013. O levantamento do índice $\mathrm{H}$ institucional ocorreu entre os meses de setembro e outubro de 2013 e foi realizado por meio do software Publish or Perish (PoP), que considera toda publicação disponível no Google Scholar (Harzing, 2007; Harzing \& Wal, 2008, 2009). Para minimizar erros relacionados ao uso do Google Scholar e do PoP, foi realizada uma revisão manual de todos os resultados obtidos.

Foram também coletadas informações relacionadas à produção dos pesquisadores por tempo de atividade (considerando-se como ponto de partida o ano no qual ocorreu a primeira publicação), gerando, a partir daí, o número de citações dividido pelo número de trabalhos e o número de citações por tempo de atividade. Os resultados são apresentados na seção Segunda dimensão: impacto sobre a ciência.

\section{Procedimentos metodológicos da investigação relacionada à repercussão na mídia}

A mídia de negócios não apenas reflete a agenda executiva, como tem ainda a capacidade de influenciá-la e amplificar novos fenômenos e ideias de gestão (Wood \& Paula, 2006, 2008). Tal mídia frequentemente utiliza especialistas - professores, consultores e pesquisadores - para apoiar a apreciação dos temas por ela tratados. Esses profissionais emprestam sua voz de autoridade, provendo análises e novas perspectivas. Além disso, eventualmente os textos jornalísticos citam ou apresentam trabalhos realizados por pesquisadores. Dessa forma, a mídia de negócios pode operar como um veículo para a disseminação do conhecimento em Administração, facilitando a conexão entre agentes de geração de conhecimento (nas instituições de pesquisa) e agentes de apropriação e utilização do conhecimento (nas empresas e em outras organizações).

Este trabalho buscou sistematizar as citações das escolas de Administração associadas aos programas analisados e as citações de seus pesquisadores e suas pesquisas em veículos selecionados da mídia de negócios. Deve-se observar que as citações encontradas e contabilizadas nesta frente de pesquisa referem-se às instituições, aos seus professores e aos seus pesquisadores, porém não exclusivamente a professores e pesquisadores de seus NDPs.

Foram escolhidos cinco veículos, reconhecidos como os mais tradicionais, relevantes e com circulação mais representativa no Brasil: o jornal O Estado de S. Paulo (Estadão), com circulação de aproximadamente 270 mil exemplares por dia, de segunda-feira a domingo; o jornal Folha de S. Paulo (Folha), com circulação de aproximadamente 290 mil exemplares por dia, de segunda-feira a domingo; o jornal Valor Econômico (Valor), com circulação média de 60 mil exemplares por dia, de segundafeira a sexta-feira; a revista Exame, de periodicidade quinzenal, com circulação de cerca de 150 mil exemplares por edição; e a revista Época Negócios, de periodicidade mensal, com circulação de aproximadamente 150 mil exemplares por edição.

O período de análise dos textos dos jornais e revistas foi de um ano, de agosto de 2011 a julho de 2012. O levantamento compreendeu a leitura de todos os textos das edições e o registro de todas as citações identificadas. Foram analisados 424 textos de 53 edições do Caderno Economia \& Negócios do Estadão, 156 textos de 52 edições do Caderno de Negócios da Folha, 1326 textos de 51 edições do 
Caderno Empresas do Valor, 504 textos de 24 edições da revista Exame e 264 textos de 11 edições da revista Época Negócios. A busca de citações foi restrita à área de Administração. Uma vez identificados, os textos com citações eram listados, identificando-se o veículo, a data, a instituição citada e o professor ou pesquisador citado. Os resultados, fruto de análise estatística descritiva simples, são apresentados na seção Terceira dimensão: repercussão na mídia de negócios.

\section{Resultados}

Esta seção apresenta os resultados das três frentes de investigação, relacionadas respectivamente aos impactos relativos à formação de pesquisadores, à ciência e à repercussão na mídia.

\section{Primeira dimensão: formação de pesquisadores}

A Tabela 1 apresenta os resultados obtidos. A FEA-USP foi a instituição que mais formou doutores no período estudado, sendo também a instituição que apresentou a mais alta taxa de doutores egressos por professor. Considerando somente o período de 2008 a 2011, para o qual há dados consolidados disponíveis de todo o País (CAPES, 2013), os quatro programas estudados responderam por $49 \%$ da formação de doutores em Administração de Empresas no Brasil.

Tabela 1

Formação de Doutores

\begin{tabular}{|c|c|c|c|c|c|}
\hline & $\begin{array}{l}\text { CEPEAD- } \\
\text { UFMG }\end{array}$ & FEA-USP & $\begin{array}{l}\text { FGV- } \\
\text { EAESP }\end{array}$ & $\begin{array}{l}\text { PPGA- } \\
\text { UFRGS }\end{array}$ & TOTAL \\
\hline Nota CAPES (triênio 2007-2009) & 6 & 7 & 6 & 7 & - \\
\hline $\begin{array}{l}\text { Número de professores no NDP do } \\
\text { programa em } 2013\end{array}$ & 28 & 56 & 33 & 39 & 156 \\
\hline $\begin{array}{l}\text { Número de egressos no período } \\
\text { 2008-2012 }\end{array}$ & 38 & 199 & 110 & 85 & 432 \\
\hline $\begin{array}{l}\text { Relação número de } \\
\text { egressos/número de professores }\end{array}$ & 1,4 & 3,6 & 3,3 & 2,2 & 2,8 \\
\hline $\begin{array}{l}\text { Egressos que atuam na instituição } \\
\text { de origem }\end{array}$ & $5 \%$ & $7 \%$ & $18 \%$ & $11 \%$ & $10 \%$ \\
\hline $\begin{array}{l}\text { Egressos que seguiram carreira } \\
\text { acadêmica }\end{array}$ & $82 \%$ & $88 \%$ & $84 \%$ & $76 \%$ & $84 \%$ \\
\hline $\begin{array}{l}\text { Egressos que atuam em instituição } \\
\text { nota } 6 \text { ou } 7\end{array}$ & $5 \%$ & $12 \%$ & $27 \%$ & $12 \%$ & $15 \%$ \\
\hline $\begin{array}{l}\text { Egressos que seguiram carreira } \\
\text { empresarial e acadêmica }\end{array}$ & $21 \%$ & $21 \%$ & $26 \%$ & $18 \%$ & $22 \%$ \\
\hline $\begin{array}{l}\text { Egressos que seguiram apenas } \\
\text { carreira empresarial }\end{array}$ & $13 \%$ & $7 \%$ & $9 \%$ & $11 \%$ & $9 \%$ \\
\hline $\begin{array}{l}\text { Egressos que atuam em Estado } \\
\text { diferente daquele no qual } \\
\text { estudaram }\end{array}$ & $11 \%$ & $22 \%$ & $28 \%$ & $25 \%$ & $23 \%$ \\
\hline
\end{tabular}

As quatro instituições apresentam percentuais similares de doutores que seguiram carreira acadêmica. A FGV-EAESP foi a instituição que teve mais doutores atuando em instituições com nota 6 
ou 7, o que é parcialmente explicado por um percentual relativamente alto de doutores egressos na FGVEAESP que se tornaram professores dessa mesma instituição.

Por outro lado, é oportuno observar o percentual de doutores que seguiram carreira empresarial e acadêmica. Somado ao percentual de doutores egressos que seguiram exclusivamente carreira empresarial, o percentual varia de $28 \%$ (FEA-USP) a $35 \%$ (FGV-EAESP), o que é significativo, pois indica que, além de formar pesquisadores, as instituições analisadas também estão formando profissionais para atuarem nas empresas e em outras organizações. Tais profissionais estão, pode-se supor, levando o conhecimento adquirido e sua capacidade analítica para além do ambiente acadêmico.

Finalmente, é relevante comentar o significado dos indicadores apresentados nesta seção em termos de impacto social. Primeiro, deve ser registrado, embora seja óbvio, que as instituições com maior capacidade de formação de doutores geram potencialmente maior impacto social. Segundo, cabe observar que os indicadores referentes ao percentual de doutores egressos que seguiram carreira acadêmica e ao percentual de doutores egressos que atuam em Estados diferentes daquele no qual estudaram são apenas informativos. De fato, não há como julgar se os percentuais encontrados são satisfatórios ou insatisfatórios, pois tanto a carreira acadêmica quanto a carreira empresarial constituem caminhos válidos, podendo gerar impacto social, ainda que sejam de naturezas diferentes; adicionalmente, tanto a atuação local quanto a atuação em outro Estado são válidas em termos de impacto social.

No entanto, o caráter informativo não reduz a importância de conhecer os indicadores, pois eles podem balizar reflexões orientadas por questões tais como: Deveríamos fomentar ainda mais a presença de egressos em empresas, de modo a levar conhecimento mais profundo e inovador sobre gestão até elas? Ou deveríamos, ao constatar um percentual já alto de egressos que seguem carreira executiva, restringir a entrada nos programas a candidatos que efetivamente apresentem perfil acadêmico, de modo a preservar a missão original, de formação de professores e pesquisadores? Ou ainda, não poderíamos atrair mais alunos de outros Estados, de modo a formá-los e incentivá-los a desenvolver, em seus Estados de origem, novos polos de pesquisa, orientados para as necessidades locais? Ou deveríamos concentrar esforços em nosso Estado, de modo a contribuir de maneira mais efetiva com o desenvolvimento de seus quadros superiores?

\section{Segunda dimensão: impacto sobre a ciência}

A Tabela 2 apresenta os resultados dos quatro programas analisados: o número de professores, o índice $\mathrm{H}$ institucional, a média das médias (de cada professor) de citações por trabalho, a média das médias (de cada professor) de citações por ano e a média das médias (de cada professor) do tempo de atividade.

Tabela 2

Indicadores de Impacto dos Programas

\begin{tabular}{lccccc}
\hline Programa & $\begin{array}{c}\text { Número de } \\
\text { professores }\end{array}$ & $\begin{array}{c}\text { Índice H } \\
\text { institucional }\end{array}$ & $\begin{array}{c}\text { Média de } \\
\text { citações dos } \\
\text { professores/ } \\
\text { trabalho }\end{array}$ & $\begin{array}{c}\text { Média de } \\
\text { citações dos } \\
\text { professores/ } \\
\text { ano }\end{array}$ & $\begin{array}{c}\text { Tempo médio } \\
\text { de atividade } \\
\text { dos professores } \\
\text { (anos) }\end{array}$ \\
\hline CEPEAD-UFMG & 28 & 25 & 3 & 9 & 20 \\
FEA-USP & 56 & 63 & 5 & 16 & 26 \\
FGV-EAESP & 33 & 60 & 7 & 26 & 22 \\
PPGA-UFRGS & 39 & 37 & 4 & 14 & 19 \\
\hline
\end{tabular}


Os resultados apontam os programas da FEA-USP e da FGV-EAESP como aqueles com maior índice $\mathrm{H}$ institucional. O programa da FGV-EAESP possui, ainda, o maior índice (médias das médias) de citações por trabalho e o maior índice (média das médias) de citações por ano. Os programas da FEAUSP e da FGV-EAESP são os mais antigos do Brasil. Esses programas, especialmente o da FEA-USP, são os que apresentam maior tempo médio de atividade dos pesquisadores. Conforme aponta Burrell (2007), o índice $\mathrm{H}$ favorece pesquisadores com mais tempo de atividade, já que tende a crescer à medida que aumenta o tempo de atividade de pesquisa e publicação dos pesquisadores.

Dois outros fatores devem ser considerados na análise desses resultados. Primeiro, o efeito da localização: os programas de FEA-USP e da FGV-EAESP localizam-se na região com maior concentração de pesquisadores do País, o que favorece as redes de cooperação, o que, por sua vez, favorece o impacto (Capobiango, Silveira, Zerbat, \& Mendes, 2011). Segundo, essas duas instituições publicam periódicos renomados no campo da Administração de Empresas: a Revista de Administração da Universidade de São Paulo (RAUSP) e a Revista de Administração de Empresas (RAE), os quais se encontram entre os mais antigos do Brasil no campo da Administração e são classificados pelo sistema Qualis como A2.

Tal condição também pode contribuir para o impacto dos respectivos programas. Isso ocorre porque os editores costumam ser professores das instituições responsáveis pela publicação do periódico, e por isso trabalham juntos aos colegas da instituição, o que facilita a comunicação sobre questões relacionadas à linha editorial e fóruns especiais, além de potencializar o pertencimento às mesmas redes de colaboração. Note-se que, na RAE, $100 \%$ do comitê de política editorial e $16 \%$ do corpo editorial científico estão ligados à FGV-EAESP (RAE, 2014). Na RAUSP, no volume 48, foram publicados 57 artigos de 154 autores. Desses, 17,5\% estavam ligados à FEA-USP (RAUSP, 2014). Assim, mesmo que tais periódicos adotem medidas contra práticas endógenas (Wood \& Chueke, 2008), persiste o efeito positivo sobre o impacto de suas respectivas instituições.

\section{Terceira dimensão: repercussão na mídia de negócios}

A Tabela 3 apresenta o número de textos analisados por veículo da mídia, o número de textos com citações e os percentuais aproximados das citações das instituições pesquisadas, de seus pesquisadores e de suas pesquisas por veículo. Nota-se uma grande diferença entre veículos: enquanto a Folha cita referências acadêmicas, sejam pesquisas científicas realizadas por escolas de Administração ou declarações de pesquisadores dessas mesmas escolas, em $26,9 \%$ de seus textos, o Valor as cita em apenas $0,5 \%$ de seus textos.

Tabela 3

\section{Citações por Veículo}

\begin{tabular}{lccc}
\hline Jornais e revistas & Textos analisados & Textos com citações & Percentual \\
\hline Folha de S. Paulo & 156 & 42 & $26,9 \%$ \\
Época Negócios & 264 & 14 & $5,3 \%$ \\
O Estado de S. Paulo & 424 & 16 & $3,8 \%$ \\
Exame & 504 & 8 & $1,6 \%$ \\
Valor Econômico & 1.326 & 6 & $0.5 \%$ \\
\hline Total & $\mathbf{2 . 6 7 4}$ & $\mathbf{8 6}$ & $\mathbf{3 , 2 \%}$ \\
\hline
\end{tabular}

A Tabela 4 apresenta a frequência de citações por veículo e por instituição. Observa-se que a FGV-EAESP é a instituição mais citada, seguida pela ESPM-SP e pelo INSPER. A FGV-EAESP é uma da mais antigas instituições brasileiras de ensino e pesquisa em Administração. Supõe-se que, com o 
tempo, tenha desenvolvido relações com a mídia, passando a ser procurada por jornalistas para conceder entrevistas e dar suas opiniões sobre assuntos da área de Negócios.

Tabela 4

Citações por Instituição

\begin{tabular}{lccccccc}
\hline Instituição & $\begin{array}{c}\text { Folha de S. } \\
\text { Paulo }\end{array}$ & $\begin{array}{c}\text { Época } \\
\text { Negócios }\end{array}$ & $\begin{array}{c}\text { O Estado de } \\
\text { S. Paulo }\end{array}$ & Exame & $\begin{array}{c}\text { Valor } \\
\text { Econômico }\end{array}$ & Citações & Percentagem \\
\hline FGV-EAESP & 12 & 5 & 4 & 4 & 2 & 27 & $31,4 \%$ \\
ESPM - SP & 6 & 1 & 6 & 2 & 0 & 15 & $17,4 \%$ \\
INSPER & 8 & 4 & 2 & 1 & 0 & 15 & $17,4 \%$ \\
FEA-USP & 6 & 1 & 0 & 0 & 2 & 9 & $10,5 \%$ \\
FGV-EBAPE & 3 & 0 & 2 & 0 & 0 & 5 & $5,8 \%$ \\
Outras ${ }^{(a)}$ & 7 & 3 & 2 & 1 & 2 & 15 & $17,4 \%$ \\
\hline Total & 42 & 14 & 16 & 8 & 6 & 86 & $100,0 \%$ \\
\hline
\end{tabular}

Nota. $\left({ }^{a}\right)$ Outras instituições citadas: Fundação Dom Cabral, Mackenzie, PUC-MG, Anhembi-Morumbi, FECAP, FAAP e ESPM-RJ.

O levantamento realizado permitiu constatar que, embora a mídia de negócios analisada tenha gerado, no período de um ano, um número expressivo de textos (2.674), apenas 3,2\% deles mencionaram como fontes pesquisadores de escolas de Administração ou pesquisas realizadas no âmbito dessas instituições. Tal percentual é baixo, considerando-se que praticamente qualquer tema tratado pela mídia de negócios poderia beneficiar-se da opinião de um especialista acadêmico. De fato, diversos dos temas mais populares na mídia de negócios - por exemplo: inovação, sustentabilidade, logística, gestão de pessoas, fusões e aquisições, e competitividade - são também temas de pesquisas conduzidas no âmbito dos programas de pós-graduação das escolas de Administração.

Além disso, constatou-se que, entre as 86 citações identificadas, apenas 25 estão associadas a professores e pesquisadores dos NDPs das instituições pesquisadas, o que representa apenas $29 \%$ do total de citações. O percentual é baixo, refletindo a distância entre a mídia e negócios e os professores e pesquisadores dos NDPs.

\section{Discussão}

Esta pesquisa focou três dimensões de impacto social no âmbito dos principais programas brasileiros de pós-graduação: a formação de doutores, o impacto na ciência e a repercussão na mídia. Os resultados obtidos sugerem a existência de oportunidades para o aumento do impacto social. Tais oportunidades são apresentadas a seguir.

\section{Oportunidades para aumento da formação de doutores}

Em relação ao impacto relacionado à formação de doutores, constatou-se considerável variação de porte e capacidade de formação entre os programas pesquisados. O programa da FEA-USP, o maior entre os analisados, com 56 professores em 2013, formou 199 doutores de 2008 a 2012, apresentando uma relação de 3,6 egressos por professor. O programa da CEPEAD-UFMG, o menor entre os analisados, com 28 professores em 2013, formou 38 doutores de 2008 a 2012, apresentando uma relação de 1,4 egresso por professor. Naturalmente, devem-se evitar comparações simplistas, que não considerem o histórico recente, as especificidades dos programas nem a quantidade de mestres egressos. 
Entretanto, os números sugerem que há oportunidade para crescimento e para aumento da relação entre egressos e professores.

Uma segunda oportunidade diz respeito à regionalização da formação de doutores. Os quatro programas analisados situam-se nas regiões Sul e Sudeste do Brasil, sendo três deles na região Sudeste (dos quais dois na cidade de São Paulo). A aceleração de programas interinstitucionais (os Dinters) pode representar uma oportunidade para ampliar a formação de doutores além das regiões mencionadas. Naturalmente, há de se cuidar, em qualquer iniciativa de expansão, dos aspectos relacionados à qualidade da formação oferecida, envolvendo tanto as disciplinas ministradas quanto o desenvolvimento das teses.

Uma terceira oportunidade, relacionada ao percentual representativo de egressos que seguem carreira empresarial, refere-se à possibilidade de criar doutorados profissionalizantes em Administração de Empresas (DBAs), programas voltados à formação de profissionais para atuação em empresas e outras organizações, oferecendo-lhes conteúdos e oportunidades de reflexão mais profundos. Banerjee e Morley (2013) comentam o crescimento dos doutorados profissionalizantes nos últimos 20 anos e argumentam que os DBAs foram desenvolvidos como resposta à crescente crítica acerca da reduzida relevância da pesquisa conduzida no âmbito dos doutorados acadêmicos (PhDs) para a prática gerencial. Os autores apresentam uma lista (não exaustiva) de 72 DBAs existentes na Austrália, Reino Unido e Estados Unidos.

O Brasil não tem, até o momento, DBAs, porém vem fomentando o desenvolvimento de mestrados profissionais em administração (MPAs). Os MPAs foram criados no Brasil a partir da década de 1990. Ribeiro (2005) argumenta que a criação de mestrados profissionais (em todos os campos de conhecimento) é sustentada por três constatações: (a) a demanda da sociedade, além das instituições de ensino e pesquisa, por formação mais qualificada; (b) o encaminhamento de parte significativa dos mestres e doutores formados para empregos não relacionados ao ensino superior; e (c) o fato de não haver preconceito em relação à transferência de conhecimento para as empresas e o mercado. O mesmo argumento e as mesmas constatações são válidos para o DBA. Tal curso tem potencial para estabelecer uma ponte sólida entre conhecimento científico e realidade organizacional, gerando, assim, impacto social.

\section{Oportunidades para aumento do impacto na ciência}

Em relação ao impacto na ciência, contatou-se também considerável variação entre os programas pesquisados. A FEA-USP e a FGV-EAESP apresentaram índices $\mathrm{H}$ de 63 e 58, respectivamente, enquanto o PPGA-UFRGS e o CEPEAD-UFMG apresentaram índices $\mathrm{H}$ de 37 e 25 , respectivamente. Além disso, uma análise complementar, realizada individualmente com os pesquisadores que compõem os NDPs desses programas, revelou forte heterogeneidade dentro dos próprios programas. Há, em todos eles, um número significativo de professores com índice $\mathrm{H}$ abaixo de 5 , variando de $18 \%$ a $32 \%$ do NDP.

Isso significa que tais pesquisadores têm, no máximo, cinco artigos que foram citados ao menos cinco vezes cada um, o que pode ser considerado um patamar baixo, tanto em termos absolutos como diante das referências apontadas por Mingers (2008). Naturalmente, tal constatação deve ser vista com cautela e analisada à luz das condições gerais dos programas brasileiros, ainda em desenvolvimento, e complementada com a análise de outros indicadores de produção científica. Ainda assim, trata-se inquestionavelmente de um ponto de atenção. Deduz-se, portanto, que há, nesse aspecto, uma oportunidade para aperfeiçoamento dos NDPs das instituições analisadas.

O impacto na ciência pode ser também maximizado com medidas já conhecidas, tais como a participação em redes internacionais de pesquisa e a consolidação das linhas de pesquisa, com o fomento à pesquisa cooperativa, fundada em projetos de médio e longo prazos, orientados para temas relevantes para as empresas e a sociedade. 
Finalmente, é preciso promover uma reflexão em relação ao sistema de avaliação da pósgraduação brasileira, que tem sido alvo de sucessivas críticas, entre as quais se destaca aquela referente à orientação para a produção - medida pelo número de artigos publicados em periódicos qualificados e não para o impacto - medido pela citação dos artigos por outros artigos (veja Alcadipani, 2011; Bertero, Vasconcelos, Binder, \& Wood, 2013; Rocha-e-Silva, 2009). É oportuno observar que a questão da avaliação acadêmica tem sido objeto de debate também fora do Brasil, frequentemente envolvendo a questão da ampliação das métricas de medição (Adler \& Harzing, 2009; Aguinis, Shapiro, Antonacopoulou, \& Cummings, 2014; Aguinis, Suárez-González, Lannelongue, \& Joo, 2012).

\section{Oportunidades para aumento da repercussão na mídia}

Em relação ao impacto relacionado à presença na mídia, há boas razões para promover a aproximação entre a mídia de negócios e as escolas de Administração. De fato, algumas instituições já contam com serviços de assessoria de imprensa, visando gerenciar de modo profissional tal interface. Tais assessorias têm papel relevante na divulgação de cursos e de outras atividades de ensino. Cumprem também o objetivo de fortalecimento institucional da marca e da reputação das instituições. No entanto, seu papel na disseminação do conhecimento gerado por meio de pesquisas científicas é menos conhecido.

A pesquisa realizada revelou que, nos cinco veículos investigados, que se encontram entre os principais jornais e revistas de negócios do Brasil, apenas 86 textos, entre 2.674 (representando 3,2\%) citavam pesquisadores ou trabalhos científicos. Há, portanto, uma oportunidade para as instituições e ensino e pesquisa de Administração ocuparem maior espaço na mídia de negócios. Isso pode se materializar tanto na disseminação de pesquisas que possam ser de interesse de empresas e da comunidade como um todo quanto pela presença de pesquisadores, analisando e comentando fenômenos relevantes do mundo corporativo.

Deve-se observar que o aumento do espaço ocupado na mídia pode gerar impactos positivos para os diversos agentes envolvidos: por um lado, pode beneficiar as empresas e a sociedade, com uma visão supostamente neutra, consistente e bem informada sobre os fenômenos e temas em foco; e, por outro lado, pode beneficiar os próprios pesquisadores, pela maior exposição e aproximação ao seu objeto de estudo, e as instituições, pela valorização de sua marca e pelo fortalecimento de sua reputação.

\section{Conclusão}

Esta seção final apresenta uma breve síntese do estudo realizado. Registra também limitações do trabalho, indicando direções para futuros estudos sobre a questão do impacto social do conhecimento.

\section{Síntese e contribuições do estudo}

Este artigo insere-se no esforço de reflexão sobre o impacto social do conhecimento gerado na academia, o que inclui os meios para sua transmissão à sociedade. Situa-se também no contexto da avaliação dos programas de pós-graduação stricto sensu no Brasil. Foi tomada como ponto de partida a perspectiva segundo a qual a avaliação do impacto social da produção científica deve ser multidimensional (Santos, 2003). Realizou-se, então, um estudo exploratório que utilizou um modelo simplificado de impacto social com três dimensões: formação, ciência e repercussão na mídia (Figura 1). Quatro programas brasileiros de pós-graduação em Administração foram pesquisados, baseados na FGV-EAESP, na FEA-USP, no PPGA-UFRGS e no CEPEAD-UFMG.

Este artigo traz duas contribuições para o estudo do impacto social do conhecimento no Brasil: primeiro, contribui para a discussão do tema no âmbito dos programas de pós-graduação em Administração de Empresas no Brasil; e, segundo, apresenta alguns levantamentos inéditos sobre 
variáveis de impacto social, relacionadas aos doutores egressos, ao fator H dos NDPs e à repercussão na mídia.

O artigo traz, ainda, contribuições práticas para a gestão dos programas de pós-graduação, pois apresenta conceitos e indicadores que podem ser utilizados para orientar decisões e ações para o aumento do impacto social. Por exemplo, os indicadores sobre a formação de doutores podem ser utilizados para orientar esforços para eventual expansão dos programas; o índice $\mathrm{H}$ pode ser utilizado para balizar decisões acerca da renovação dos NDPs; e os resultados apurados sobre repercussão na mídia podem orientar diretrizes voltadas para a aproximação entre escolas e veículos de comunicação, visando fomentar a disseminação de novas pesquisas e novos conhecimentos.

\section{Limitações do estudo e sugestões para futuras pesquisas}

Este estudo apresenta algumas limitações, que poderão ser tratadas em futuras pesquisas. A primeira limitação refere-se à escolha dos programas, que se restringiu àqueles com nota 6 e 7 segundo o sistema CAPES, na avaliação realizada no triênio 2007-2009. Futuras pesquisas poderão ampliar o número de programas estudados, podendo, assim, fornecer um retrato mais completo da realidade brasileira. A ampliação do estudo poderá também comprovar, ou refutar, o pressuposto adotado, segundo o qual o resultado informado pelo sistema de avaliação da CAPES pode ser tomado como predição do impacto social.

A segunda limitação refere-se à escolha e operacionalização das dimensões de análise. O modelo (Figura 1) utilizado é uma simplificação do modelo proposto por Santos (2003), coerente com o objetivo (exploratório) do estudo e com o objeto de estudo: os programas de pós-graduação em Administração de Empresas.

Entretanto, futuras pesquisas poderão ampliar consideravelmente o escopo de investigação. No âmbito da formação de pesquisadores, futuras pesquisas poderão, por exemplo, focar a produção científica e o fator $\mathrm{H}$ dos doutores egressos; poderão também investigar os mestrandos egressos. No âmbito do impacto sobre a ciência, futuras pesquisas poderão incluir outros indicadores de impacto, além do fator $\mathrm{H}$, tais como a presença em periódicos de topo, locais e internacionais. No âmbito da repercussão na mídia, futuras pesquisas poderão investigar mídias regionais e mídias especializadas (por exemplo, revistas dedicadas a temas tais como varejo, recursos humanos e logística). Com isso, teríamos um retrato mais completo do impacto social. Futuras pesquisas poderão também procurar identificar evidências qualitativas de impacto social. Por exemplo, pesquisas que facilitem decisões visando à reorganização de cadeias produtivas; ou estudos que levem à alteração de práticas de gestão de pessoas; ou, ainda, estudos que levem a mudanças em práticas gerenciais, visando ao aumento da competitividade.

\section{Referências}

Adler, N. J., \& Harzing, A.-W. (2009). When knowledge wins: transcending the sense and nonsense of academic rankings. Academy of Management Learning \& Education, 8(1), 72-95. doi: 10.5465/AMLE.2009.37012181

Aguinis, H., Shapiro, D. L., Antonacopoulou, E. P., \& Cummings, T. G. (2014). Scholarly impact: a pluralistic conceptualization. Academy of Management Learning \& Education, 13(4), 623-639. doi: 10.5465/amle.2014.0121

Aguinis, H., Suárez-González, I., Lannelongue, G., \& Joo, H. (2012). Scholarly impact revisited. Academy of Management Perspectives, 26(2), 105-132. doi: 10.5465/amp.2011.0088 
Alcadipani, R. (2011). A academia e a fábrica de sardinhas. Organizações \& Sociedade, 18(57), 345348.

Banerjee, S., \& Morley, C. (2013). Professional doctorates in management: toward a practice-based approach to doctoral education. Academy of Management Learning \& Education, 12(2), 173-193. doi: 10.5465/amle.2012.0159

Bennis, W. G., \& O'Toole, J. (2005). How business schools lost their way. Harvard Business Review, 83(5), 96-104.

Bertero, C. O., Vasconcelos, F. C., Binder, M. P., \& Wood, T., Jr. (2013). Produção científica brasileira em administração na década de 2000. Revista de Administração de Empresas, 53(1), 12-20. doi: $10.1590 / \mathrm{S} 0034-75902013000100002$

Bornmann, L., \& Daniel, H.-D. (2007). What do we know about the H index? Journal of the American Society for Information Science and Technology, 58(9), 1381-1385. doi: 10.1002/asi.20609

Braun, T., Glanzel, W., \& Schubert, A. (2006). A Hirsch-type index for journals. Scientometrics, 69(1), 169-173. doi: 10.1007/s11192-006-0147-4

Burrell, Q. (2007). Hirsch index or Hirsch rate? Some thoughts arising from Liang's Data. Scientometrics, 73(1), 19-28. doi: 10.1007/s11192-006-1774-5

Bush, V. (1945). Science, the endless frontier: a report to the president. Washington, DC: U. S. Government Printing Office.

Capobiango, R. P., Silveira, S. F. R., Zerbato, C., \& Mendes, A. C. A. (2011). Análise das redes de cooperação científica através do estudo das coautorias dos artigos publicados em eventos da Anpad sobre avaliação de políticas públicas. Revista de Administração Pública, 45(6), 18691990. doi: 10.1590/S0034-76122011000600012

Chia, R. (1996). Teaching paradigm shifting in management education: university business schools and the entrepreneurial imagination. Journal of Management Studies, 33(4), 409-428. doi: 10.1111/j.1467-6486.1996.tb00162.x

Chia, R. (1999). A 'rhizomic' model of organizational change and transformation: perspective from a metaphysics of change. British Journal of Management, 10(3), 209-227.

Comissão Europeia. (2010). Assessing Europe's university-based research: expert group on assessment of university-based research. Luxembourg: Publications Office of the European Union. Recuperado de https://ec.europa.eu/research/sciencesociety/document_library/pdf_06/assessing-europe-university-based-research_en.pdf

Coordenação de Aperfeiçoamento de Pessoal de Nível Superior. (2013). Documento de área 2013. Recuperado http://www.capes.gov.br/images/stories/download/avaliacaotrienal/Docs_de_area/Administra\% C3\%A7\%C3\%A3o_doc_area_e_comiss\%C3\%A3o_16out.pdf

Estébanez, M. E. (2003). Impacto social de la ciencia y la tecnología: estrategia para su análisis. Recuperado de http://www.ricyt.org/manuales/doc_view/117-impacto-social-de-la-ciencia-y-latecnologia-estrategias-para-su-analisis

Garfield, E. (1999). Journal impact factor: a brief review. Canadian Medical Association Journal, 161(8), 979-980.

Garrett-Jones, S. (2000). International trends in evaluating university research outcomes: what lessons for Australia. Research Evaluation, 9(2), 115-124. doi: 10.3152/147154400781777278 
Glänzel, W. (2008). Seven myths in bibliometrics about facts and fiction in quantitative science studies. Collnet Journal of Scientometrics and Information Management, 2(1), 9-17. doi: 10.1080/09737766.2008.10700836

Grey, C., \& French, R. (1996). Rethinking management education: an introduction. In R. French \& C. Grey (Eds.), Rethinking management education (pp. 1-17). Sage: London.

Grey, C., Knights, D., \& Willmott, H. (1996). Is a critical pedagogy of management possible? In R. French \& C. Grey (Eds.), Rethinking management education (pp. 94-110). Sage: London.

Gross, P. L. K., \& Gross, E. M. (1927). College libraries and chemical education. Science, 66(1713), 385-389. doi: 10.1126/science.66.1713.385

Guisado, Y. M., Cabrera, F. M. S., \& Cortés, J. N. (2010). Aproximaciones a la evaluación del impacto social de la ciencia, la tecnología y la innovación. ACIMED, 21(2), 161-183.

Harzing, A.-W. (2007). Publish or perish. Recuperado em 7 julho, 2011, de http://www.harzing.com/pop.htm

Harzing, A.-W., \& Wal, R. van der (2008). Google Scholar as a new source for citation analysis. Ethics in Science and Environmental Politics, 8(61), 61-73. doi: 10.3354/esep00076

Harzing, A.-W., \& Wal, R. van der (2009). Google Scholar H-Index for journals: an alternative metric to measure journal impact in economics \& business? Journal of the American Society for Information Science and Technology, 60(1), 41-46. doi: 10.1002/asi.20953

Hirsch, J. E. (2005). An index to quantify an individual's scientific research output. Proceedings of the National Academy of Sciences of the United States of America, 102(46), 16569-16572. doi: 10.1073/pnas.0507655102

Lima, G. M. R., \& Wood, T., Jr. (2014). The social impact of research in business and public administration. Revista de Administração de Empresas, 54(4), 458-463. doi: 10.1590/S0034759020140410

Macias-Chapula, C. A. (1998). O papel da informetria e da cienciometria e sua perspectiva nacional e internacional. Ciência da Informação, 27(2), 134-140. doi: 10.1590/S0100-19651998000200005

Mingers, J. (2008). Measuring the research contribution of management academics using the Hirsch Index [Working Paper Series $n^{\circ}$ 162]. Kent Business School, University of Kent. Recuperado de http://kar.kent.ac.uk/25453/1/Hirsch1_sent_Web_Version.pdf

Mintzberg, H. (2004). Managers not MBAs: a hard look at the soft practice of managing and management development. San Francisco: Berret-Koehler.

O Estado de S. Paulo. (2013, dezembro 23). Alckmin nomeia Zago como novo reitor da USP. Recuperado em 27 dezembro, 2013, de http://www.estadao.com.br/noticias/vidae,alckminnomeia-zago-como-novo-reitor-da-usp, $1112556,0 . h t m$

Palomares-Montero, D., García-Aracil, A., \& Castro-Martínez, E. (2008). Evaluación de las instituciones de educación superior: revisión bibliográfica de sistema de indicadores. Revista Española de Documentación Científica, 31(2), 205-229. doi: 10.3989/redc.2008.v31.i2.425

Pfeffer, J., \& Fong, C. T. (2002). The end of business schools? Less success than meets the eye. Academy of Management Learning \& Education, 1(1), 78-95. doi: 10.5465/AMLE.2002.7373679

Raan, A. F. J. van (2006). Comparison of the Hirsch-index with standard bibliometric indicators and with peer judgment for 147 chemistry research groups. Scientometrics, 67(3), 491-502. doi: 10.1556/Scient.67.2006.3.10 
Revista de Administração da Universidade de São Paulo. (2014). Informações editoriais. Recuperado em 23 junho, 2014, de http://www.rausp.usp.br/informacoes-editoriais.asp

Revista de Administração de Empresas. (2014). Corpo editorial. Recuperado em 23 junho, 2014, de http://rae.fgv.br/RAE/institucional/Corpo-Editorial

Reynolds, M. (1997). Towards a critical management pedagogy. In J. Burgoyne \& M. Reynolds (Eds.), Management learning: integrating perspectives in theory and practice (pp. 312-338). London: Sage.

Ribeiro, R. J. (2005). O mestrado profissional na política atual da Capes. Revista Brasileira de Pósgraduação, 2(4), 8-15.

Rocha-e-Silva, M. (2009). O novo Qualis, ou a tragédia anunciada. Clinics, 64(1), 1-4. doi: $10.1590 / \mathrm{S} 1807-59322009000100001$

Rubin, R. S., \& Dierdorff, E. C. (2009). How relevant is the MBA? Assessing the alignment of required curricula and required managerial competencies. Academy of Management Learning \& Education, 8(2), 208-224. doi: 10.5465/AMLE.2009.41788843

Ruiz, M. A., Greco, O. T., \& Braile, D. M. (2009). Fator de impacto: importância e influência no meio editorial, acadêmico e científico. Revista Brasileira de Cirurgia Cardiovascular, 24(3), 273-278. doi: 10.1590/S0102-76382009000400004

Sanderson, M. (2008). Revisiting h measured on UK LIS and IR academics. Journal of the American Society for Information Science and Technology, 59(7), 1184-1190. doi: 10.1002/asi.20771

Santos, R. N. M. (2003). Produção científica: por que medir? O que medir? Revista Digital de Biblioteconomia e Ciência da Informação, 1(1), 22-38.

The Association to Advance Collegiate Schools of Business. (2008). Impact of research task force. Final report of the AACSB International. Tampa, Florida: AACSB International.

The Association to Advance Collegiate Schools of Business. (2012). Impact of research: a guide for business schools. Tampa, Florida: AACSB International.

The Association to Advance Collegiate Schools of Business. (2013). Eligibility procedures and accreditation standards for business accreditation. Tampa, Florida: AACSB.

Vieira, B. F. S. V. (2013b, dezembro, 27). Novo reitor terá de abrir mais portas da USP. O Estado de S. Paulo. Recuperado em 27 dezembro, 2013, de http://www.estadao.com.br/noticias/impresso,novo-reitor-tera-de-abrir-mais-portas--da-usp,1112693,0.htm

Walker, R., Alexander, J., Barros, B., Cochrane, M., Guzman, E., Geuna, A., Hoppe, U., Iemma, R., Sandor, S., Zeini, S., Lawson, C., \& Göhnert, T. (2011). Requirements document and specification of conceptual model. Recuperado de http://sisob.lcc.uma.es/repositorio/deliverables/SISOBD22.pdf

Walker, R., Alexander, J., Barros, B., Guzman, E., Cochrane, M., Geuna, A., Hppe, U., Iemma, R., Sandor, S., Zeni, S., del-Campo, J., \& Ramos, G. (2011). Literature review. Recuperado de http://sisob.lcc.uma.es/repositorio/deliverables/SISOB-D2.1_Literature_Review.pdf

Willmott, H. (1994). Management education: provocations to a debate. Management Learning, 25(1), 105-136. doi: 10.1177/1350507694251008 
Wood, T., Jr., \& Chueke, G. V. (2008). Ranking de produção científica em administração de empresas no Brasil. Revista de Administração Mackenzie, 9(4), 13-31. doi: 10.1590/S167869712008000400003

Wood, T., Jr., \& Paula, A. P. P. de (2006). A mídia especializada e a cultura do management. Organizações \& Sociedade, 13(38), 91-105. doi: 10.1590/S1984-92302006000300006

Wood, T., Jr., \& Paula, A. P. P. de (2008). Pop-management literature: popular business press and management culture in Brazil. Canadian Journal of Administrative Sciences, 25(3), 185-200. doi: $10.1002 /$ cjas. 71

\section{Dados dos Autores}

Thomaz Wood Jr.

Av. Nove de Julho, 2029, 01313-902, São Paulo, SP, Brasil. E-mail: thomaz.wood@fgv.br

Caio César Medeiros Costa

Av. Nove de Julho, 2029, 01313-902, São Paulo, SP, Brasil. E-mail: caiocesq@ hotmail.com

Giovanna de Moura Rocha Lima

Av. Nove de Julho, 2029, 01313-902, São Paulo, SP, Brasil. E-mail: gimrlima@ gmail.com

Rosana Córdova Guimarães

Av. Nove de Julho, 2029, 01313-902, São Paulo, SP, Brasil. E-mail: ro_cordova@yahoo.com.br 